\title{
EXTRAGALACTIC BACKGROUNDS: COMMON FEATURES AND NEW WORK ON INTRACLUSTER LIGHT
}

\author{
R. B. Partridge \\ Haverford College \\ Haverford, PA 19041 USA
}

\begin{abstract}
I examine some common features of several extragalactic backgrounds, beginning with the best studied of all backgrounds, the $3 \mathrm{~K}$ microwave radiation, then continuing on to the newly discovered submillimeter flux and the near infrared (IR) background. Both the total surface brightness and fluctuations (or anisotropies) in the brightness of these backgrounds are considered. Some implications for cosmology and for astrophysics are mentioned.
\end{abstract}

\section{INTRODUCTION}

In this review, I consider the low energy end of the electromagnetic spectrum, that is photon energies of $\sim 0.001$ to $10 \mathrm{eV}$. The reader will soon see that many of the points I consider have been raised by others here. Allow me to begin with the best studied of all extragalactic backgrounds, and the one I know best, the $3 \mathrm{~K}$ microwave radiation.

\section{AN EXAMPLE OF A WELL-STUDIED EXTRAGALACTIC BACKGROUND: THE 3 K COSMIC BACKGROUND RADIATION}

The results of recent observational and theoretical work on the cosmic microwave background radiation (CBR) are thoroughly reviewed by De Zotti (1989, this volume). Rather than repeat aspects of his paper, let me use some of the earliest observational work on the CBR as an illustrative example of how studies of other extragalactic backgrounds might proceed. I believe there are useful parallels with other possible backgrounds at shorter wavelengths.

Soon after the discovery of the CBR 25 years ago (Penzias and Wilson, 1965; Dicke et al., 1965), it was recognized that the radiation had to pass two important tests to establish its cosmological origin: spectrum (a cosmic background would be blackbody) and isotropy (a cosmic background would be uniform and not correlated with local sources of radio emission, such as the plane of the Galaxy). By the late 1960's, it was clear that the radiation had passed both tests. The spectrum was consistent with a blackbody of $\mathrm{T} \sim 3 \mathrm{~K}$, including a hint of a Wien turnover at $3 \mathrm{~mm}$ wavelength (Wilkinson, 1967); and the radiation was isotropic to better than 1\% on large scales (Conklin and Bracewell, 1967; Partridge and Wilkinson, 1967).

Attention then came to focus on the possibility that Penzias and Wilson had observed the sum of microwave emission from many faint, discrete, radio sources at cosmological distances (Wolfe and Burbidge, 1969). If so, the CBR ought to be anisotropic on small angular scales, essentially because of Poisson scatter in the number of sources in the beam of the radio telescope used to observe it (Smith and Partridge, 1970). Careful searches for such small-scale anisotropy (reviewed by De Zotti, 1989, this volume) have failed to find any at the $\sim 10^{-4}$ level. The limits are now good enough to show that if discrete sources were responsible for the entire CBR, they would have to be incredibly numerous to keep the fluctuation level low - certainly more numerous than ordinary galaxies. Of course, it remains true that discrete sources could still produce some small fraction of the $3 \mathrm{~K}$ background and could dominate fluctuations in it (Danese, De Zotti, and Mandolesi, 1983). But the bulk of the CBR must be 
diffuse, consistent with a cosmological origin in a hot Big Bang.

Those familiar with questions surrounding the X-ray background (XRB), reviewed by Setti (1989, this volume), will recognize parallels with that field. Can a good part or all of the XRB be produced by discrete sources, quasars, AGN's, etc.? Can such a discrete source model explain the observed spectrum and, in particular, the fine-scale isotropy of the XRB (see Hamilton and Helfand, 1987)?

\section{THE SUBMILLIMETER EXCESS}

The same questions may be asked about the submillimeter flux recently measured by Matsumoto and his colleagues from Nagoya and Berkeley (1989, this volume). Is this excess flux observed in the Wien region of the 2.7-2.8 K CBR in fact a new cosmological background, or is it rather a distortion of the CBR spectrum? Once again, both the spectrum and the isotropy of the submillimeter radiation may provide useful tests.

Let us consider some of the models that have been suggested for this excess flux. As just noted, these models may be divided into two classes: distortions of the CBR spectrum (with no new emission mechanisms required) and new cosmic backgrounds (requiring an emission mechanism and an energy source).

In the first category is inverse Compton distortion produced either before or after the CBR decouples from matter at redshift $\mathrm{z} \sim 1000$. As is well known, inverse Compton scattering off hot electrons preserves photon number but increases photon energy. Hence a very characteristic distortion is produced (Figure 1). The degree of distortion is parameterized by (Sunyaev and Zel'dovich, 1972)

$$
y=\int \frac{k T_{e}}{m_{e} c^{2}} n_{e} c \sigma_{T h} d t,
$$

where $T_{e}, n_{e}$, and $m_{e}$ are the electron temperature, number density, and mass, and $\sigma_{T h}$ is the Thomson scattering cross section. To fit the observations of Matsumoto et al. (1988), y must be $\sim 0.03$ (e.g., Hayakawa et al., 1987; Smoot et al., 1988). A detailed examination of the spectrum, however, reveals problems with the fit. First, the predicted increase of photon flux in the Wien region requires a decrease in the Rayleigh-Jeans region; the expected temperature at $\lambda \geq 1 \mathrm{~cm}$ is close to $2.6 \mathrm{~K}$, not the $2.74 \mathrm{~K}$ observed (Smoot et al., 1985; Johnson and Wilkinson, 1987). In addition, the fit to the most precise measurements of the CBR flux, at $1.2 \mathrm{~cm}$ (Johnson and Wilkinson, 1987) and $0.26 \mathrm{~cm}$ (Meyer and Jura, 1985; Crane et al., 1986) is not good, and the higher values for $\mathrm{T}$ at $0.26 \mathrm{~cm}$ determined from recent $\mathrm{CN}$ measurements discussed at IAU Symposium 139 (Bowyer and Leinert, 1989) do not help the agreement. It is important to recognize that the problems raised by spectral measurements confront any inverse Compton model, no matter what the source of the energy is.

Other suggested models rely on the generation of additional submillimeter photons, not just scattering. One is the radiative decay of low mass particles, perhaps one of the sort suggested as "dark matter" candidates. There are several problems with the direct generation of the entire submillimeter flux by such a process (see, e.g., Hayakawa et al., 1987). One is the spectrum, again. Radiative decay of the generic type $A \rightarrow B+\gamma$ will produce photons of a fixed energy, i.e., line emission. The cosmological redshift will smear out a spectral line into a continuum, but one which does not fit the observations of Matsumoto et al. (1988). Other models in this category, including an indirect means of supplying the needed energy by particle decay, are discussed here by Wang and Field (1989, this volume). 


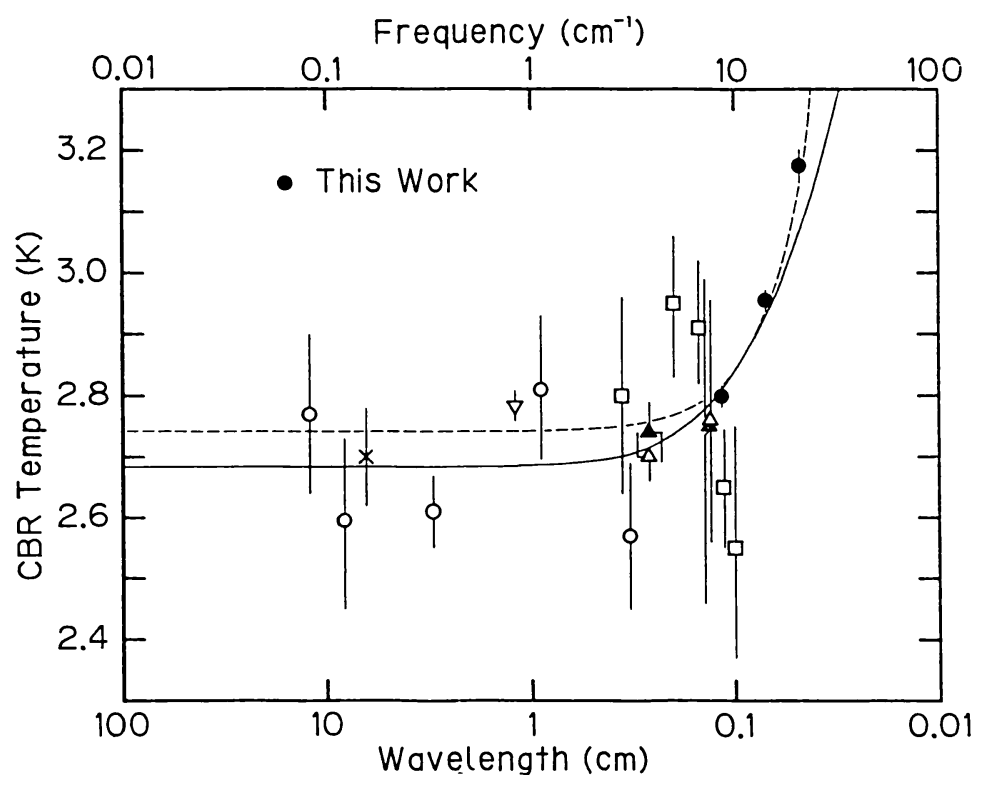

Figure 1. The distortion of the cosmic microwave background spectrum resulting from inverse Compton scattering (from Matsumoto et al., 1988). The solid line is an inverse Compton model (in this case, with $y=.02$ ); the dashed line a superposition of the CBR and emission from dusty galaxies. Results of other measurements shown for comparison are Peterson et al. (1985) (open squares), Meyer and Jura (1985) (open triangles), Crane et al. (1986) (filled triangles), Mandolesi et al. (1986) (cross), Smoot et al. (1985, 1987) (open circles), Johnson and Wilkinson (1987) (open inverted triangle).

Let me now turn to a final model - thermal reemission by dust in galaxies at a sufficiently large redshift to place the radiation at 300-1000 $\mu$ m (Hayakawa et al., 1987; Bond, Carr and Hogan, 1989). This model, too, is constrained to some degree by the observed spectrum. (The emitting galaxies must be at a large redshift, $z \sim 10-20$, and the bulk of the emission must occur in a narrow redshift range in that broader interval to explain the sharply peaked submillimeter emission; see further discussion by De Zotti, 1989, this volume.)

A more stringent test of the dusty galaxy model may be provided by searches for smallscale anisotropies in the submillimeter background - we are back, in a sense, to the argument of Wolfe and Burbidge (1969) that the summed emission from many discrete sources may produce the observed background radiation. If so, as Bond, Carr, and Hogan (1989) among others have noted, it will be anisotropic because of the fluctuations in the number of sources in the beam of the instrument used to observe it. The result is an anisotropy with an amplitude proportional to $\theta^{-1}$, where $\theta$ is the scale probed.

As yet, there is only a single direct measurement of anisotropy in the $300-1000 \mu \mathrm{m}$ region, the work of Melchiorri et al. (1981) and Fabbri et al. (1982) on a scale of $6^{\circ}$. That angular scale is too large to test the dusty galaxy model severely. Recently, as part of a program to detect quasars at $\lambda=1.3 \mathrm{~mm}$, Kreysa and Chini (1989) were able to put a limit on temperature fluctuations on an angular scale of $11^{\prime \prime}$ : this is $\Delta T / T \leqq 2.6 \times 10^{-4}$, corresponding to intensity fluctuations of $\Delta I / I \leq 10^{-3}$. This observational limit on the fine-scale anisotropy implies that, if discrete sources make up the submillimeter background, their number must considerably exceed the number of galaxies per steradian (Bond, Carr, and Hogan, 1989). The work of Kreysa and Chini is currently the most sensitive constraint we have on dusty galaxy or 
other discrete source models for the background found by the Berkeley-Nagoya group (Matsumoto et al., 1988).

Even smaller angular scales may be probed using microwave interferometry or aperture synthesis. Unfortunately, such observations have so far been made at $\lambda \geqslant 2 \mathrm{~cm}$, where the $3 \mathrm{~K}$ radiation dominates the submillimeter flux. Nevertheless, $2 \mathrm{~cm}$ observations at $\theta=5 " 4$ (Hogan and Partridge, 1989) may be used to set limits on $\Delta I / I$ of the submillimeter flux on this angular scale. We do so by assuming that the $3 \mathrm{~K} \mathrm{CBR}$ itself is perfectly isotropic, so any anisotropy at $\lambda=2 \mathrm{~cm}$ is due entirely to the small component of the $2 \mathrm{~cm}$ flux contributed by the long wavelength tail of the submillimeter background. How large that contribution is and, consequently, how stringent a limit our measurements allow, depend on the emissivity of the dust in the high redshift galaxies. If we take $\eta \propto v^{\alpha}$, we find $\Delta I / I<0.2$ for $\alpha=1$ at $\theta=5 " 4$ (no useful limit results from our work if $\alpha=2$ ).

It is worth mentioning that limits on the large-scale anisotropy of the submillimeter flux may be set in a somewhat similar way, by ascribing all the dipole or quadrupole isotropy measured at a wavelength $\lambda$ to the contribution of the submillimeter background at that wavelength. For instance, Partridge and Lahav (1988) used the $3.3 \mathrm{~mm}$ results of Lubin, Melese, and Smoot (1983) to show that the submillimeter flux has a dipole moment $<4 \%$ or $20 \%$ for $\alpha=1$ or 2 . The quadrupole component is smaller, $<0.5 \%$ or $<2 \%$. Tighter limits on the anisotropy of the submillimeter excess may be set by using higher frequency measurements of the CBR (see Halpern et al., 1988). These limits rule out a Galactic origin for the submillimeter excess.

\section{NEAR-INFRARED BACKGROUND}

Let us now turn to the high-frequency end of the infrared spectrum. As pointed out twenty years ago, the formation of the first generation of stars in the Universe, whether in young galaxies (Partridge and Peebles, 1967) or not (Eichler and Solinger, 1976; Thorstensen and Partridge, 1975), will produce a copious ultraviolet flux. If this radiation is trapped in dusty young galaxies, the energy will emerge in the far-IR, as noted above. If it escapes, on the other hand, it will create visible or near-IR extragalactic light (EGL), depending on the redshift of formation of the stars. This topic is reviewed masterfully by Mattila (1989, this volume); see also the paper by Toller (1989, this volume).

Searches for visible or near-IR, isotropic, cosmological backgrounds are notoriously difficult, as Mattila points out. Both atmospheric emission and Galactic starlight (direct and scattered) present major obstacles in the visible; a variety of clever techniques has been used (Mattila, 1976; Dube, Wilkes, and Wilkinson, 1979; Boughn, Saulson, and Uson, 1986) to circumvent them. In the 1-5 $\mu \mathrm{m}$ range, the Galactic foreground may be somewhat less, but atmospheric emission remains or even increases as we leave the visible.

Matsumoto, Akiba and Murakami (1988) have made a pioneering effort to measure EGL in the near-IR above the atmosphere by mounting their instrument on a sounding rocket. Figure 2 reproduces their results. At $\sim 3-5 \times 10^{-12} \mathrm{~W} / \mathrm{cm}^{2} \mu \mathrm{m}$ sr, or $\lambda I_{\lambda} \sim 10^{-7} \mathrm{~W} / \mathrm{m}^{2} \mathrm{sr}$, their measured surface brightness is rather high; to generate that much flux in young galaxies would require a conversion of something like $\sim 20 \%$ of their mass to heavy elements. This result is so important that additional work is needed to confirm it. Matsumoto (1989, this volume) reports on the plans of the Nagoya group. Let me describe briefly an attempt we have underway to measure the near-IR EGL, using a different procedure.

\subsection{A Planned Balloon Experiment}

The experiment is a collaboration between the Istituto TESRE of the Italian Centro Nazionale delle Ricerche (CNR), located in Bologna, and Haverford College; the effort is led by Dr. N. 


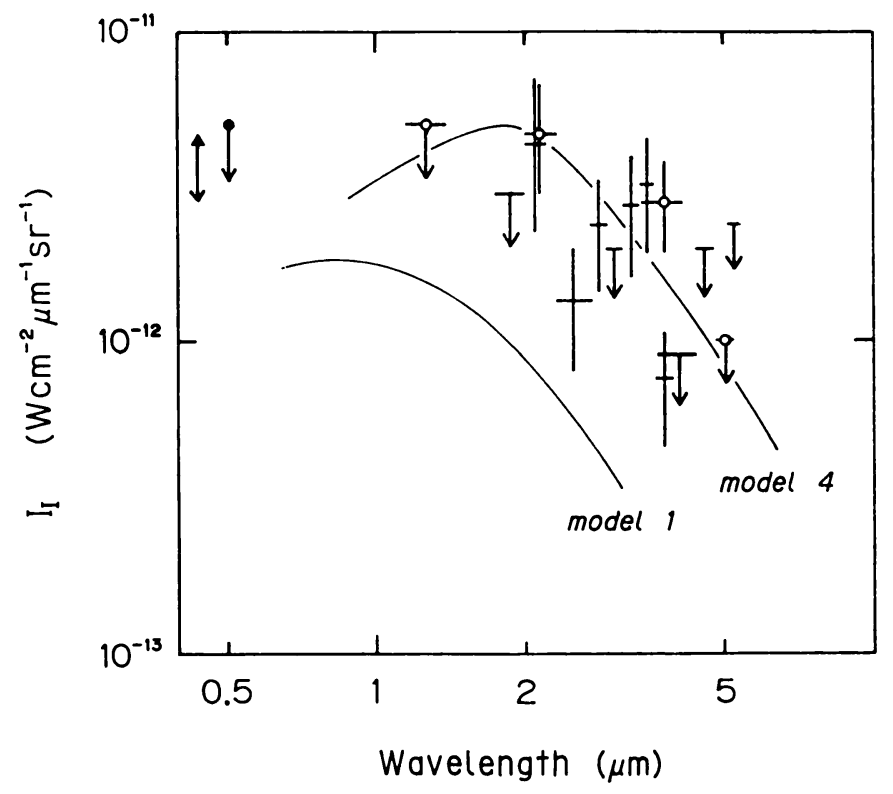

Figure 2. The near-IR rocket measurements of Matsumoto, Akiba and Murakami (1988). As noted in the text, we hope to repeat $\sim 2 \mu \mathrm{m}$ observations using a cooled, balloon-borne telescope; our goal is $I \leq 10^{-12} \mathrm{~W} / \mathrm{cm}^{2} \mu \mathrm{m}$ sr. Open circles and crosses represent the data of the wide-band and narrow-band channels, respectively. Upper limits of the extragalactic background light (EBL) at the visible band (Dube et al. 1977: filled circle; Toller 1983: filled triangle) are also shown. EBL calculated by Partridge and Peebles (1967) for two extreme cases is indicated by solid lines.

Mandolesi of TESRE, and Steve Boughn and I are involved.

We plan to fly a balloon-borne, cooled telescope of $25 \mathrm{~cm}$ diameter to make absolute measurements of EGL in the near-IR at several wavelengths in the $1.5-4.0 \mu \mathrm{m}$ band. The telescope and supporting electronics were designed and built at TESRE and other Italian institutions. As a detector, we employ a linear array of $32 \mathrm{InSb}$ diodes; the pixel scale is 1.38 on the sky, so each observation will cover a strip $44^{\prime}$ long. To reduce stray radiation, the entire telescope is cooled to $\sim 55 \mathrm{~K}$ using He gas.

A balloon flight, unlike a rocket flight, will not carry the telescope beyond the $\mathrm{OH}$ emission layer in the upper atmosphere. Thus we will devote much of our observing time to measuring residual atmospheric emission, including $\mathrm{OH}$. First, we employ a set of filters specifically centered on atmospheric emission features, including $\mathrm{OH}$ lines. Two other filters (at $-2.0-2.5 \mu \mathrm{m}$ ) were designed to avoid all strong atmospheric lines; these will provide our primary EGL measurements. Second, we will make observations at several zenith angles in order to measure residual atmospheric emission. We will also make measurements at 12 equally spaced azimuths. During a 6-8 hour flight, we will thus sample a reasonable fraction $(-50 \%)$ of the northern hemisphere. We hope these measurements will enable us to unfold (and to measure) both Galactic emission at $1.5-4.0 \mu \mathrm{m}$ and the zodiacal light.

A balloon flight is a risky proposition; so is the decision to work beneath the OH layer. We hope that the use of an array of detectors and an observing time $\sim 100$ times longer than afforded by a rocket flight will outweigh those risks. If all goes according to plan, we should be able to measure the EGL spectrum from $1.5-4.0 \mu \mathrm{m}$ at a level of $\sim 10^{-12} \mathrm{~W} / \mathrm{cm}^{2} \mu \mathrm{m}$ sr or better sensitivity. At this level, we will be testing the theoretical models listed above. 


\subsection{Near-IR Anisotropy}

This balloon flight is designed to check the spectrum of the near-IR EGL. What about complementary observations of its isotropy? Several years ago, my colleague at Haverford, Steve Boughn, realized that the near-IR background produced by newly formed galaxies would display small-scale, shot-noise anisotropies of the sort we have considered before. Boughn, Saulson, and Uson (1986) therefore set out to measure or set limits on fluctuations in the background light at $2.2 \mu \mathrm{m}$, using a ground-based telescope and detector. Their limits on intensity fluctuations on scales of 10-300" lie approximately an order of magnitude below the total $2 \mu \mathrm{m}$ intensity reported by the Nagoya group, that is, at $\sim 4-9 \times 10^{-13} \mathrm{~W} / \mathrm{cm}^{2} \mu \mathrm{m}$ sr. Thus, these ground-based observations can set comparably stringent limits on theories of galaxy formation.

\section{A REMARK ON EGL AND THE RADIATIVE DECAY OF PARTICLES}

I have already had occasion to mention the possibility that the radiative decay of low mass particles (such as neutrinos of nonzero mass) might contribute to the EGL. Let me now consider how EGL measurements may be used to constrain the fundamental properties of these particles, such as their mass and decay lifetime. Since the "dark matter" needed to bind galaxies and clusters may consist of such particles, any information on their properties would be of considerable value (see Knapp and Cormendy 1989 for details).

We begin by supposing that these particles - which I will refer to as neutrinos of nonzero mass $m_{v}$ even though other possibilities can be and have been imagined - can decay radiatively into some other massless or low-mass particle $L$. It is then easy to show that the photon energy resulting from such a decay is

$$
E_{\gamma}=\frac{1}{2}\left[\frac{m_{v}^{2}-m_{L}^{2}}{m_{v}}\right] c^{2} \approx \frac{1}{2} m_{v} c^{2},
$$

where the latter approximation holds for $m_{v} \gg m_{L}$. Note that line emission will result.

For such particles to play any useful role in cosmology now (e.g., binding galaxies or clusters gravitationally), their lifetime must be $\geq H_{0}^{-1}$ or $3 \times 10^{17}$. The presence and radiative decay of such particles therefore implies a cosmological background, with a characteristic wavelength depending on $m_{v}$ (Kimble, Bowyer, and Jakobsen, 1981; Shipman and Cowsik, 1981).

Now let us consider the possibility that such particles are aggregated. Specifically, it has been suggested (e.g., by Cowsik and McClelland, 1973, and Schramm and Steigman, 1981) that massive neutrinos might supply the "missing mass" to bind clusters of galaxies. If so, phase space arguments (Tremaine and Gunn, 1979; Schramm and Steigman, 1981) suggest that $m_{v} \sim 4 \mathrm{eV}$. Thus the radiative decay of these particles would produce EGL in the visible. Furthermore, if the radiatively decaying particles are clustered, the EGL they produce is easier to detect since we can make differential, rather than absolute, measurements of the light they produce. Thus limits on EGL in clusters may be used to set limits on the radiative decay lifetime of massive neutrinos. If the mass-to-light ratio $(M / L)$ of the intergalactic matter is expressed in physical units (gm erg), it may be shown that the $1 /$ e decay time $\tau$ is $\tau=4.5 \times 10^{20}$ $(M / L)$. 


\section{LIMITS ON M/L OF INTERGALACTIC LIGHT IN CLUSTERS}

With two colleagues, Alan Kiplinger (now at the University of Colorado) and Matthew Bershady (now at the University of Chicago), I measured the EGL in eight clusters of galaxies of different Bautz-Morgan types, richnesses, and redshifts.

These observations were made nearly a decade ago. Despite some uncertainty in the calibration, I present them here because of the current interest in searches for intergalactic light (IGL) in clusters of galaxies and elsewhere. For details see an informal report circulated by Partridge, Kiplinger, and Bershady (1989).

In our search for light concentrated in clusters but not associated with the individual galaxies in the clusters, we performed area photometry using an early charge-coupled device (CCD) camera borrowed from Dave Wilkinson's group at Princeton University. The CCD had $100^{2}$ pixels; the pixel scale was $3.3^{\prime \prime} \times 4.4^{\prime \prime}$. We took CCD photographs in three spectral bands, $\mathrm{V}, \mathrm{r}$, and a truncated $\mathrm{i}$ band, cut off at $\lambda \sim 8500 \AA$ to avoid atmospheric OH lines. Although our filters are fairly wide $(\Delta \lambda / \lambda \sim 25 \%)$, our measurements may still be used to set limits on the radiative decay lifetime, $\tau$.

We made observations of clusters at galactic latitudes above $45^{\circ}$. The clusters were selected also to be free of bright foreground stars; we accepted only those clusters in which there were no stars brighter than $13 \mathrm{~m} .5$. From the list of potential candidates, we selected our eight final candidates to have a range of Abell richness classes and of Bautz-Morgan types.

We took a number of steps to control systematic errors. First, we offset the cluster center alternately to the east and west of the center of our 5.5 $\times 7.3 \mathrm{CCD}$ field. This was done to avoid any possible spurious radial symmetry introduced by vignetting, etc. We thus had two independent pictures of each cluster in each band. Each cluster picture was divided, pixel-bypixel, by a sky picture taken of a nearby area also free of bright stars, and then renormalized using the average sky brightness. We measured the point spread function of a star and used the results to correct our pictures for the light of remaining stars and galaxies in the field; we dropped any data for which this correction exceeded $1 \%$ of the night sky brightness.

In each of the resulting pictures, we located by eye twenty to thirty $5 \times 5$ pixel $(-350$ $\left.\operatorname{arcsec}{ }^{2}\right)$ areas apparently free of stars or galaxies. These samples were made at different distances $r$ from the nominal cluster center.

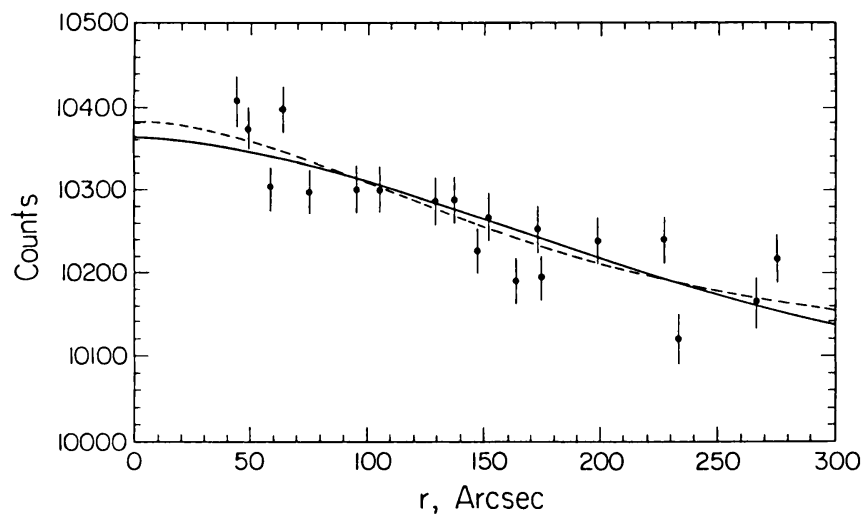

Figure 3. A plot of surface brightness in the $r$ band as a function of distance from the center of Abell cluster 2065. The two model profiles are the King profile (solid line), and a more centrally concentrated profile appropriate for a cluster bound by degenerate neutrinos (dashed line). 
The intensity of these samples as a function of $r$ was then plotted (Figure 3). At this stage, we checked for slope across the CCD field; in some cases, when it was small, we made a linear correction for it. We then compared these graphs with various models for the profile of the intergalactic light. The first of these is based on the King $(1966,1972)$ profile, which assumes that galaxies trace the mass distribution in the cluster. Another, essentially a triangle profile in density, assumes that the cluster mass was dominated by fully degenerate neutrinos or other fermions (e.g., Shipman and Cowsik, 1981). For each model, the integrated light was calculated.

We then attempted to correct these figures for IGL for the light of faint galaxies in the cluster, below our detection thresholds of $20.0,20 \mathrm{~m} .0$, and $19^{\mathrm{m}} .0 \mathrm{in} \mathrm{V}, \mathrm{r}$, and i. These corrections were small $(-0.1-0.5$ of the observed light, except for Abell cluster 2645 , the most distant cluster). These corrected results appear in Table 1.

TABLE 1. Corrections for the Light of Faint Galaxies and Final Values for Intergalactic Light

\begin{tabular}{|c|c|c|c|c|}
\hline Cluster & Filter & $\begin{array}{c}\text { FGL in each band } \\
\mathrm{erg} / \mathrm{sec} \times 10^{44}\end{array}$ & $\begin{array}{l}\text { Uncorrected } \\
\text { Luminosity }\end{array}$ & 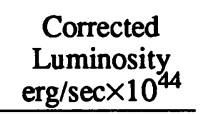 \\
\hline \multirow{2}{*}{566} & $\mathbf{r}$ & 3.3 & $18.9 \pm 5.8$ & $15.6 \pm 5.9$ \\
\hline & i & 2.6 & $9.3 \pm 6.2$ & $6.7 \pm 6.2$ \\
\hline \multirow[t]{3}{*}{665} & V & 5.6 & $12.1 \pm 8.4$ & $+6.5 \pm 8.5$ \\
\hline & $\mathbf{r}$ & 5.7 & $12.7 \pm 8.3$ & $+7.0 \pm 8.4$ \\
\hline & $\mathbf{i}$ & 4.4 & $2.7 \pm 5.0$ & $-1.7 \pm 5.1^{*}$ \\
\hline \multirow[t]{3}{*}{2029} & V & 3.8 & $-0.4 \pm 8.7$ & $-4.2 \pm 8.8$ \\
\hline & $\mathbf{r}$ & 3.7 & $+6.9 \pm 6.6$ & $+3.2 \pm 6.7$ \\
\hline & i & 2.8 & $+19.3 \pm 5.5$ & $+16.5 \pm 5.5$ \\
\hline 2065 & $\mathbf{r}$ & 3.6 & $16.1 \pm 6.8$ & $12.5 \pm 16.9$ \\
\hline \multirow[t]{3}{*}{2255} & V & 1.5 & $3.6 \pm 9.3$ & $2.1 \pm 9.3$ \\
\hline & $\mathbf{r}$ & 1.3 & $4.9 \pm 10.5$ & $3.6 \pm 10.5$ \\
\hline & $\mathrm{i}$ & 1.1 & $3.0 \pm 4.1$ & $1.9 \pm 4.1$ \\
\hline \multirow[t]{3}{*}{2256} & V & 1.6 & $10.2 \pm 5.3$ & $8.6 \pm 5.3$ \\
\hline & $\mathbf{r}$ & 1.5 & $3.6 \pm 1.4$ & $2.1 \pm 1.4$ \\
\hline & i & 1.2 & $8.1 \pm 2.1$ & $6.9 \pm 2.1$ \\
\hline \multirow[t]{3}{*}{2645} & v & 9.0 & $-4.3 \pm 5.0$ & $-13.3 \pm 5.5$ \\
\hline & $\mathbf{r}$ & 12.9 & $+0.6 \pm 3.0$ & $-12.3 \pm 4.4^{*}$ \\
\hline & $\mathbf{i}$ & 10.3 & $+5.7 \pm 2.9$ & $-4.6 \pm 3.9 *$ \\
\hline \multirow[t]{3}{*}{2670} & V & 1.0 & $-0.4 \pm 12.6$ & $-1.4 \pm 12.6$ \\
\hline & $\mathbf{r}$ & 0.9 & $-1.1 \pm 6.2$ & $-2.0 \pm 6.2$ \\
\hline & i & 0.7 & $+11.0 \pm 5.0$ & $+10.3 \pm 5.0$ \\
\hline
\end{tabular}

In several clusters, in one or more color band, we have tentative evidence for IGL. Only in Abell cluster 2256 do we see significant IGL in all three bands. There were no consistent trends connecting IGL with cluster type or richness.

By making an assumption about the spectrum of the light we observe, namely, that it matches the spectral energy distribution of E galaxies given by Yoshii and Takahara (1988), we may use our samples in three color bands to estimate the total bolometric intergalactic luminosities of these clusters. That value is typically $20 \%$ of the total light (taken from Dressler, 1978) of the clusters. These figures in turn can be used to calculate $M / L$ ratios for clusters of known mass (e.g., from Dressler, 1978). We find $M / L_{\text {bolo }} 1500$ for the IGL, in physical units of $\mathrm{gm} \mathrm{s} \mathrm{erg}^{-1}$ (or 3000 in solar units).

To set limits on the radiative decay lifetime of neutrinos, we need to look at $M / L$ ratios in each color band, not $M / L_{\text {bolo }}$, since line emission is expected. For a King profile, $M / L$ is 
as large as $4 \times 10^{4}$ in the $\mathrm{V}, \mathrm{r}$, and $\mathrm{i}$ bands; for the less extended triangle profile appropriate for degenerate fermions, $M / L$ is typically a factor $2-6$ more. We thus find $1 / \mathrm{e}$ decay lifetimes in excess of $1-2 \times 10^{25}$ (or $\sim 10^{8} \mathrm{H}_{o}^{-1}$ !), if such particles bind clusters of galaxies (see Bershady and Partridge, 1989, for details). These results represent a modest improvement on limits on $\tau$ set by observations of a single nearby cluster, Coma, by Shipman and Cowsik (1981), and cover a wider range of $m_{\mathrm{v}}$. Note that our limits apply only to radiative decay lifetimes.

The same observations may be used to set constraints on a different kind of intergalactic matter: stars tidally stripped from the member galaxies of the cluster. For instance, if we take $10,000,5,000$, and 3,000 as the color temperature of these stars, we find that $<0.02 \%,<0.3 \%$ and $<4 \%$ of the total cluster mass can be made up of stars. To test the more interesting mass in clusters, near-IR measurements are more valuable (see Boughn, 1989, this volume).

Our observations are not as useful as narrow-band filter measures for detecting line emission from gas in cooling flows (see Cowie et al., 1983). They may, however, be used to test the possibility raised by Mattila (1977) that light scattering by intergalactic dust in clusters might produce or add to IGL in clusters. Since we generally see $<20 \%$ of the total cluster light, an approximate limit of 0.2 may be placed on the optical depth due to scattering in the optical. Our work, of course, places no limit on the optical absorption due to such dust, which other work (Karachentsev and Lipovetskii, 1969) suggests may have an optical depth -0.12 in the red.

Acknowledgments - Much of my work mentioned here was supported by grants from the U.S. National Science Foundation over the years. The IR balloon project is supported largely by the CNR of Italy; among the members of the TESRE group are S. Cortiglioni, G. Giannini, N. Mandolesi, G. Morigi, and G. Ventura. Finally, I thank the organizers of IAU Symposium 139 for an opportunity to return to Heidelberg.

\section{REFERENCES}

Bershady, M. A., and Partridge, R. B. 1989, in preparation.

Bond, J. R., Carr, B. J., and Hogan, C. J. 1989, Ap. J., submitted.

Boughn, S. 1989, Proc. IAU 139, Galactic and Extragalactic Background Radiation, ed. S. Bowyer and Ch. Leinert (Dordrecht: Kluwer Academic Publisher).

Boughn, S. P., Saulson, P. T., and Uson, J. M. 1986, Ap. J., 301, 17.

Bowyer, S. and Leinert, Ch., eds. 1989, Proc. IAU 139, Galactic and Extragalactic Background Radiation (Dordrecht: Kluwer Academic Publisher).

Conklin, E. K., and Bracewell, R. N. 1967, Phys. Rev. Letters, 18, 614.

Cowie, L. L., Hu, E. M., Jenkins, E. B., and York, D. G. 1983, Ap. J., 272, 29.

Cowsik, R., and McClelland, J. 1973, Ap. J., 180, 7.

Crane, P., Hegyi, D. J., Mandolesi, N., and Danks, A. C. 1986, Ap. J., 309, 822.

Danese, L., De Zotti, G., and Mandolesi, N. 1983, Astron. Astrophys., 121, 114.

De Zotti, G. 1989, Proc. IAU 139, Galactic and Extragalactic Background Radiation, ed. S. Bowyer and Ch. Leinert (Dordrecht: Kluwer Academic Publisher).

Dicke, R. H., Peebles, P. J. E., Roll, P. G., and Wilkinson, D. T. 1965, Ap. J., 142, 414.

Dressler, A. 1978, Ap. J., 226, 55.

Dube, R. R., Wickes, W. C., and Wilkinson, D. T. 1979, Ap. J., 232, 333.

Eichler, D., and Solinger, A. 1976, Ap. J. 203, 1.

Fabbri, R., Guidi, I., Melchiorri, F., and Natale, V. 1982, in Proceedings of the Second Marcel Grossmann Meeting on General Relativity, R. Ruffini, ed., North Holland Publ. Co.

Halpern, M., Benford, R., Meyer, S., Muehlner, D., and Weiss, R. 1988, Ap. J., 332, 596.

Hamilton, T. T., and Helfand, D. J. 1987, Ap. J., 318, 93.

Hayakawa, S., Matsumoto, T., Matsuo, H., Murakami, H., Sato, S., Lange, A. E., and Richards, P. L. 1987, Publ. Astron. Soc. Japan, 39, 941.

Hogan, C. J., and Partridge, R. B. 1989, Ap. J. (Letters), 341, L29.

Johnson, D. G., and Wilkinson, D. T. 1987, Ap. J. (Letters), 313, L1.

Karachentsev, I. D., and Lipovetskii, V. A. 1969, Sov. A. J., 12, 909. 
Kimble, R., Bowyer, S., and Jakobsen, P. 1981, Phys. Rev. Letters, 46, 80.

King, I. R. 1966, A.J., 71, 64.

King, I. R. 1972, Ap. J. (Letters), 174, L123.

Knapp and Cormendy, eds. 1989, Proc. IAU 139, Dark Matter (Dordrecht: Reidel).

Kreysa, E., and Chini, R. 1989, in Proc. of the Third ESO-CERN Symposium on Astronomy, Cosmology and Fundamental Particles, in press.

Lubin, P., Melese, P., and Smoot, G. 1983, Ap. J. (Letters), 273, L51.

Matsumoto, T., et al. 1989, Proc. IAU 139, Galactic and Extragalactic Background Radiation, ed. S. Bowyer and Ch. Leinert (Dordrecht: Kluwer Academic Publisher).

Matsumoto, T., Akiba, M., and Murakami, H. 1988, Ap. J. 332, 575.

Matsumoto, T., Hayakawa, S., Matsuo, H., Murakami, H., Sato, S., Lange, A. E., and Richards, P. L. 1988, Ap. J., 329, 567.

Mattila, K. 1976, Astron. Astrophys., 47, 77.

Mattila, K. 1977, Astron. Astrophys. 60, 425.

Mattila, K. 1989, Proc. IAU 139, Galactic and Extragalactic Background Radiation, ed. S. Bowyer and Ch. Leinert (Dordrecht: Kluwer Academic Publisher).

Melchiorri, F., Melchiorri, B., Ceccarelli, C., and Pietranera, L., 1981, Ap. J. (Letters), 250, L1.

Meyer, D. M., and Jura, M. 1985, Ap. J., 297, 119.

Partridge, R. B., and Lahav, O. 1988, Mon. Not. Roy. Astr. Soc., 235, 1.

Partridge, R. B., and Peebles, P. J. E. 1967, Ap. J., 148, 377.

Partridge, R. B., and Wilkinson, D. T. 1967, Phys. Rev. (Letters), 18, 557.

Penzias, A. A., and Wilson, R. W. 1965, Ap. J., 142, 419.

Schramm, D. N., and Steigman, G. 1981, Ap. J., 243, 1.

Setti, G. 1989, Proc. IAU 139, Galactic and Extragalactic Background Radiation, ed. S. Bowyer and Ch. Leinert (Dordrecht: Kluwer Academic Publisher).

Shipman, H. L., and Cowsik, R. 1981, Ap. J. (Letters), 247, L111.

Smith, M. G., and Partridge, R. B. 1970, Ap. J., 159, 737.

Smoot, G. F., De Amici, G., Friedman, S., Witebsky, C., Sironi, G., Bonelli, G., Mandolesi, N., Cortiglioni, S., Morigi, G., Partridge, R. B., Danese, L., and De Zotti, G. 1985, Ap. J. (Letters), 291, L23.

Smoot, G., Levin, S. M., Witebsky, C., De Amici, G., and Rephaeli, Y. 1988, Ap. J., 331, 653.

Sunyaev, R. A., and Zel'dovich, Ya. B. 1972, Comments Astrophys. Space Sci., 4, 173.

Thorstensen, J. R., and Partridge, R. B. 1975, Ap. J., 200, 527.

Toller, G. 1989, Proc. IAU 139, Galactic and Extragalactic Background Radiation, ed. S. Bowyer and Ch. Leinert (Dordrecht: Kluwer Academic Publisher).

Tremaine, S., and Gunn, J. E. 1979, Phys. Rev. Letters, 42, 407.

Wang, B., and Field, G. B. 1989, Proc. IAU 139, Galactic and Extragalactic Background Radiation, ed. S. Bowyer and Ch. Leinert (Dordrecht: Kluwer Academic Publisher).

Wilkinson, D. T. 1967, Phys. Rev. Letters, 19, 1195.

Wolfe, A. M., and Burbidge, G. R. 1969, Ap. J., 156, 345.

Yoshii, Y., and Takahara, F. 1988, Ap. J., 326, 1.

B. Wang: What are the masses you consider? How do they compare with the limits from stellar evolution models?

B. Partridge: The mass range (for neutrinos etc.) is narrow: $2-5 \mathrm{eV}$, because our observations are restricted to optical and near infrared photons. I believe the lifetimes we are discussing $\left(\approx 10^{7} H_{0}^{-1}\right)$ are much larger than those relevant to stellar evolution limits.

K. Meisenheimer: Have you thought about producing the additional light in clusters by lensing and enhancing of background objects?

B. Partridge: That is an interesting point which we have not considered. My impression is that the number and the surface brightness of the background objects we saw in Tony Tyson's very deep cluster pictures were too small to add appreciably to the cluster light. Your question will be best answered by the work Tyson and his associates have under way to produce refined and more sensitive estimates of the intergalactic light in clusters. 
A. Tyson: We have had some difficulty doing this experiment. In a program of deep imaging of rich clusters at moderate redshift, we see clear evidence of "intergalactic" diffuse cluster light. However, it has been very difficult to uniquely remove the low surface brightness wings of the cluster galaxies. Most of the brighter cluster galaxies have surface brightness exceeding $30 \mathrm{R}-\mathrm{mag} \operatorname{arcsec}^{-2}$ out to angles approximating the mean galaxy-galaxy separation. Thus, the resulting diffuse cluster light is dependent on the galaxy surface brightness model.

Y. Rephaeli: I just want to point out that measurements of diffuse intracluster light are also relevant for setting constraints on a component of gas colder than that which emits the X-rays.

J. Peebles: Have you information on the spectrum of the excess cluster light you detected; and can you test the possibility that you have detected starlight?

B. Partridge: Please remember that our data are rather rough, so we cannot extract a spectrum. We have, however, played the game of asking what fraction of the total cluster mass can reside in stars of various color temperatures - see the figures in my review.

S. Boughn: People who do cluster dynamics predict that on the order of $10 \%$ to $40 \%$ of the stars from galaxies are tidally stripped and reside in the cluster potential. The fact that Partridge detects only $5 \%$ diffuse light is, therefore, interesting.

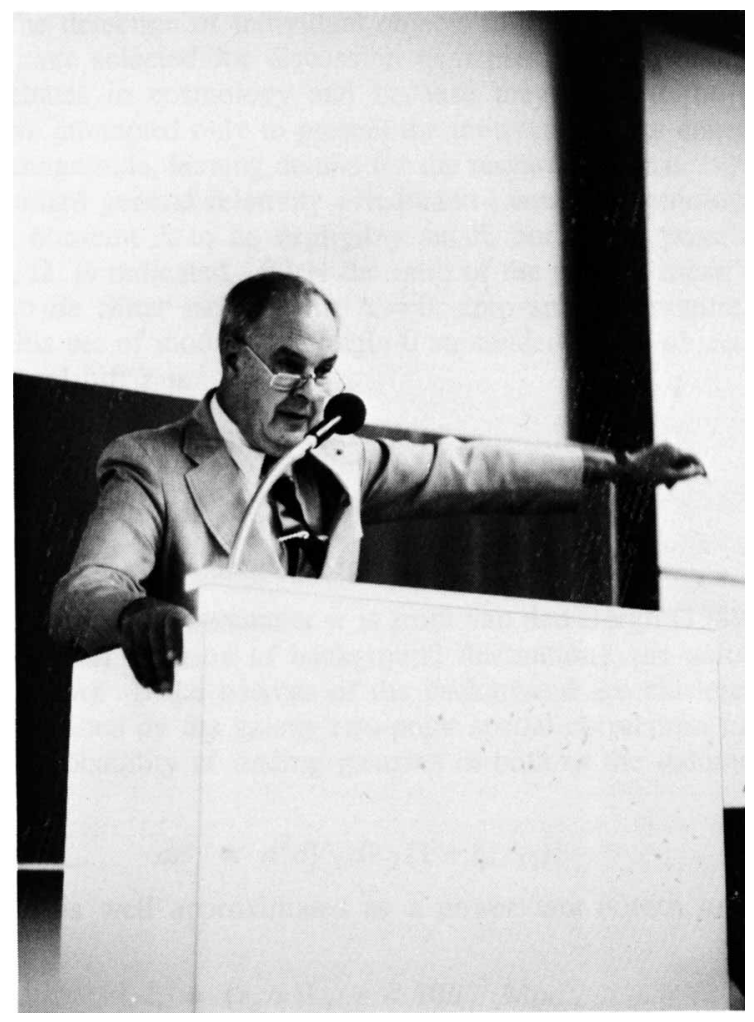

Opening address by Guido Münch 